\title{
Est-ce vraiment un "décrochage culturel ॥? Une analyse critique du concept de décrochage culturel à partir des écrits sur le choix scolaire des élèves en transition primaire- secondaire
}

\author{
Megan Cotnam \\ Université d'Ottawa
}

\section{Résumé}

Plusieurs parents ayants-droit ne choisissent pas le système scolaire francophone pour leurs enfants. De plus, même lorsque ces parents optent pour l'école primaire francophone, un nombre important d'adolescents décident, seuls ou avec leur famille, de quitter le système francophone lors de la transition vers le secondaire. Depuis quelques décennies, plusieurs chercheurs et agents d'éducation ontariens qualifient ces jeunes de « décrocheurs culturels », un terme qui semble impliquer un rejet complet de la langue et de la culture françaises. En est-il vraiment ainsi? Afin d'évaluer la pertinence de l'expression « décrochage culturel », cet article recense les écrits qui traitent du choix scolaire en tant que processus parental et familial. Cette recension révèle de nombreux facteurs d'influence dont, entre autres, le consumérisme, le statut socioéconomique, l'opinion des parents quant à l'importance de la langue française et du bilinguisme, l'influence des parents selon l'âge de l'élève, l'opinion des amis et l'insécurité linguistique. Le choix semble lié à la recherche d'un avenir meilleur et n'est pas uniquement une question de culture. Ces constats remettent en cause le bien-fondé de l'expression «décrochage culturel», d'autant plus que des conséquences négatives découlant de l'utilisation de cette expression ont déjà été notées chez certains jeunes concernés.

\section{Abstract}

A significant number of right holder parents do not choose the French-language school system for their children. Moreover, even when parents choose a French elementary school, there are many adolescents who choose, either alone or with their family, to leave the Francophone system during the transition to high school. In the last few decades, several researchers and educators from Ontario labeled francophone students who choose to attend an English school as "cultural dropouts", an expression which seems to imply a rejection of the French language and culture entirely. Is this new expression appropriate? To find some answers to this question, the following article reviews the literature regarding parents and school choice, as well as school choice as a family process. This review uncovers 
numerous factors that influence the choice process, such as socioeconomic status, consumerism, the parents' opinions regarding the importance of the French language and bilingualism, the parents' influence depending on the student's age, friends' influence and linguistic insecurity. The school choice seems to be linked to the search for a better future, and cannot be reduced to the question of culture only. These facts do not support the use of the expression "cultural dropouts", especially if one takes also into account the possible negative repercussions already noted for some of the students concerned. 


\section{Introduction}

L'école de langue française occupe une place importante dans la lutte pour la survie des langues et des cultures françaises et acadiennes en milieu minoritaire francophone au Canada (Deveau, Landry et Allard, 2006; Martel, 2001). Or, Martel (2001) indique qu'à l'échelle du pays, seulement $54,4 \%$ des enfants de parents ayants-droit fréquentent des écoles de langue française. II y a donc une proportion importante de couples ayants-droit qui ne choisit pas l'école française pour leurs enfants. En outre, même lorsque ces couples optent pour l'école primaire francophone, il y a un nombre important d'adolescents qui choisissent, seuls ou avec leur famille, de quitter le système francophone lors de la transition vers le secondaire. En Ontario, par exemple, entre la $8^{e}$ et la $9 e$ année, les écoles de langue française perdaient, en 2004, 9,6 \% de leurs effectifs, soit 632 élèves. Les écoles de langue anglaise, pour leur part, connaissaient un gain de $6,5 \%$, soit 9726 élèves, lors de cette même période de transition (Ministère de l'éducation de l'Ontario [MÉO], 2006). Ces constats montrent l'intérêt de s'interroger sur les façons non seulement d'attirer davantage d'élèves dans les écoles de langue française, mais aussi de les y inclure de telle sorte qu'ils développent un fort sentiment d'appartenance et persévèrent jusqu'à la fin de leurs études secondaires.

Selon Dalley et Saint-Onge (2008), « l'inclusion à part entière [des élèves] ne peut se faire que sur la base d'une compréhension accrue de leur réalité » (p. 139). Bien sûr, accroitre la compréhension de la réalité des élèves implique que l'on s'intéresse davantage à leur point de vue. Cependant, jusqu'à présent, relativement peu d'études (Allaire, Michaud, Boissonneault, Côté et Pounthioun, 2005; Tremblay, 2007) s'intéressent au point de vue des jeunes en ce qui concerne la qualité de leur expérience scolaire en milieu minoritaire, et celles-ci ciblent surtout le phénomène du décrochage scolaire. Si le décrochage scolaire renvoie au fait de quitter les études avant l'obtention du diplôme secondaire, certains auteurs en Ontario ont récemment établi un parallèle entre ce concept et le choix des jeunes francophones, à la fin du primaire, de quitter l'école française afin de poursuivre leurs études au niveau secondaire 
dans une école anglophone. Ils qualifient alors ce choix de « décrochage culturel ». Depuis que cette expression a été proposée, elle semble gagner en popularité parmi les chercheurs et les agents d'éducation ontariens, et même dans les médias (Allaire et al., 2005; Boissonneault, Michaud, Côté, Tremblay et Allaire, 2007; Fédération de la jeunesse franco-ontarienne [FESFO], 2006; MÉO, 2006; Michaud, Boissonneault, Côté, Tremblay et Allaire, 2008; Patrimoine canadien, 2006; Radio Canada, 2011; Tremblay. Côté, Boissonneault, Michaud et Allaire, 2007; Tremblay, 2007).

Lors d'un sondage ${ }^{1}$ sur les choix scolaires que nous avons effectué auprès d'élèves d'une école secondaire en milieu minoritaire du Centre-Sud de l'Ontario, en avril 2010, nous avons introduit le terme « décrochage culturel »à un groupe d'élèves de $8^{e}$ année. Les réactions de ces derniers se sont avérées très fortes : plusieurs n'étaient pas d'accord avec le fait qu'on utilise cette expression pour désigner un élève qui choisit de quitter l'école de langue française pour s'inscrire dans une école de langue anglaise et ne voudraient certainement pas être étiquetés de décrocheurs culturels. Lorsque nous avons demandé pourquoi, une élève a répondu : « Comment peut-on décrocher d'une partie de nous?» (Notes de terrain, 28 avril 2010). Et puisque les autres élèves ont fait signe qu'ils acquiesçaient à ce commentaire, cette expression nous est apparue particulièrement inappropriée. II nous est alors devenu apparent que celle-ci représentait une seule conséquence possible du choix scolaire d'une langue de scolarisation autre que le français : l'assimilation. De plus, les réactions des élèves nous ont convaincue que ce terme dénotait un manque de confiance et de respect envers leur choix.

À la suite de cet incident, nous nous sommes interrogée sur le bien-fondé de l'utilisation de « décrochage culturel » : à quelles réalités renvoie cette expression? S'agit-il d'un facteur en cause dans le processus de choix scolaire ou d'une conséquence du choix de poursuivre ses études dans une école anglaise? Étant donné son association au concept de décrochage scolaire, la notion de décrochage culturel n'est-elle pas trop négative? Vientelle embrouiller le tableau? Vient-elle culpabiliser les élèves? Qui plus est, selon une perspective bourdieusienne (Bourdieu, 1982; Bourdieu et Passeron, 1970), l'utilisation de ce terme revient-elle à une forme de violence symbolique imposée sur un groupe dominé d'élèves? À notre avis, le premier pas vers la rétention des effectifs dans les écoles de langue française au niveau secondaire en milieu minoritaire est de mieux comprendre le processus de choix scolaire

\footnotetext{
${ }^{1}$ Le sondage cité dans cet article a été administré dans le cadre d'un projet de recherche que nous avons mené lors de nos études de maîtrise à l'Université d'Ottawa. Cependant, puisque nous avons effectué un passage accéléré du programme de maîtrise au programme de doctorat, nous n'avons ni soumis, ni défendu une thèse de maîtrise. Ainsi, les données de ce sondage n'ont pas été rendues publiques jusqu'à maintenant. Si vous désirez plus d'informations sur les résultats du sondage en question, vous pouvez contacter l'auteure à l'adresse suivante : $\underline{\text { mcotn025@uottawa.ca. }}$
} 
que vivent les parents et les élèves eux-mêmes. Quelle est donc vraiment la contribution du décrochage culturel dans ce processus?

Ainsi, dans le cadre de cet article, nous nous attarderons d'abord aux écrits qui traitent du décrochage culturel. II s'agit de déconstruire ce concept et d'examiner quelles significations les chercheurs et les agents d'éducation ontariens accordent à cette expression. Ensuite, nous examinerons les écrits concernant le choix scolaire en tant que processus parental, d'une part, et de processus familial incluant la participation de l'enfant, d'autre part. Enfin, nous discuterons de la pertinence du concept de décrochage culturel dans le processus de choix scolaire des jeunes ontariens. Mais auparavant, il convient de décrire brièvement la méthodologie utilisée dans la recherche documentaire et d'établir le cadre d'analyse de la documentation recensée.

\section{Méthodologie de recherche documentaire et cadre d'analyse}

Mieux comprendre le phénomène de choix scolaire et obtenir une définition claire du terme « décrochage culturel » n'est pas une tâche simple. Pour ce faire, nous avons opté pour une analyse de contenu des documents existants dans le domaine académique et professionnel. Ces documents ont été répertoriés à l'aide des portails de recherche ERIC (Wilson) et Scholars Portal, deux portails qui ont accès à de nombreuses bases de données en éducation et sont accessibles sur le site web de la bibliothèque de l'Université d'Ottawa. Les mots-clés suivants ont été utilisés pour effectuer la recherche : choix scolaire/school choice, parent, élève/student, famille/family, années de transition/transition years/school transitions, dérochage/dropout, décrochage culturel/décrocheur culturel/cultural dropout. Avec ces mots-clés, plus de 40 articles scientifiques ont été repérés. Une recherche additionnelle dans Internet avec les mots clés « décrochage/décrocheur culturel », en plus de la consultation des sites de la FESFO et du MÉO, a permis d'ajouter à la liste plus d'une vingtaine de rapports de recherche, documents de politique et documents de vulgarisation supplémentaires ${ }^{1}$.

L'examen du contenu de ces documents a fait émerger plusieurs thèmes pertinents abordés dans les études sur le choix scolaire. Ces thèmes permettent de mieux cerner les données théoriques et empiriques susceptibles de supporter ou non le concept de décrochage culturel. Ainsi, les écrits recensés peuvent se classer en deux catégories principales selon qu'ils s'intéressent au processus de choix scolaire parental ou familial. Dans les écrits sur le choix parental, les thèmes représentent une variété de facteurs qui viennent influencer les parents qui s'apprêtent à choisir une école pour leur enfant tels que le statut socioéconomique, la mentalité de consommateurs et le magasinage d'écoles, la réussite scolaire des élèves, le rôle de la culture et le bilinguisme. Dans les écrits qui traitent du choix scolaire comme processus familial, les thèmes abordés incluent principalement l'influence des parents, l'influence des amis et des pairs et les facteurs linguistiques et culturels. Ces thèmes serviront de cadre d'analyse pour évaluer la pertinence de l'utilisation de l'expression « décrochage culturel », d'apparition récente dans la littérature sur le choix scolaire des enfants en milieu minoritaire francophone en particulier.

\footnotetext{
${ }^{1}$ Une version anglaise d'une partie de la recension des écrits présentée dans cet article a été publiée antérieurement. En voici la référence :

Cotnam, M. (2011). School Choice Connections around the Globe: A Critical Reading of School Choice Literature. Queen's Graduate Student Symposium Selected Papers, 5, 23-43.
} 


\section{La notion de « décrochage culturel »}

À notre connaissance, la notion de « décrochage culturel » fait son apparition au cours des années 1990. La première mention écrite de ce type de décrochage provient d'un mémoire de la FESFO présenté à la Commission royale sur l'éducation de l'Ontario en décembre 1993 (cité dans Lamoureux, 1995). Dans le cadre de ce document, le décrochage scolaire et culturel figure parmi les « sept aires critiques en éducation ». Qui plus est, Lamoureux (1995) explique que pour la FESFO,

la question du décrochage de l'école secondaire franco-ontarienne pour se diriger vers les écoles de langue anglaise est aussi importante sinon plus que le décrochage scolaire. Afin de pallier au décrochage culturel, [les membres de la FESFO] affirment que l'on doit agir simultanément sur trois volets : la vie académique, les réalités sociales et le sens d'appartenance à la communauté scolaire (p. 10).

Par la suite, on constate que le MÉO, Patrimoine canadien ainsi que plusieurs chercheurs qualifient de plus en plus les élèves francophones qui choisissent de fréquenter une école de langue anglaise de " décrocheurs culturels », une expression qui semble impliquer que ces derniers abandonnent complètement la langue et la culture françaises (Allaire et al., 2005; FESFO, 2006; Frenette et Quazi, 1997; Lamoureux, 1995; MÉO, 2006; Patrimoine canadien, 2006; Tremblay, 2007).

La plupart des écrits sur le décrochage culturel découlent d'une étude sur le décrochage scolaire, c'est-à-dire l'abandon des études secondaires avant l'obtention du diplôme (Allaire et al., 2005; Ferguson et al., 2005; Tremblay, 2007). Allaire et ses collaborateurs $(2005$, p. 20) identifient quatre catégories de jeunes qui décrochent de l'école au niveau secondaire : l'étudiant à risque (étudiants identifiés à risque de décrocher), le raccrocheur diplômé (l'élève qui reprend ses études après avoir décroché), le décrocheur général (le jeune qui abandonne ses études secondaires), et le décrocheur culturel (celui qui transfère d'une langue de scolarité à une autre). Ainsi, le " décrochage culturel » peut aisément être perçu comme un problème important étant donné son association au phénomène du décrochage scolaire dont les causes sont souvent reliées à une expérience scolaire jugée insatisfaisante par l'élève et dont les conséquences économiques et sociales, largement documentées, sont des plus sérieuses. Même si Allaire et al. (2005) ne concluent pas que les élèves « décrocheurs culturels » choisissent l'école anglaise parce qu'ils rejettent la francophonie, ces auteurs affirment que ces jeunes choisissent une telle école pour s'assurer ce qu'ils perçoivent comme un meilleur avenir : travailler en anglais, étudier en anglais au niveau postsecondaire, et ainsi de suite. Cette équipe de chercheurs a publié plusieurs articles sur le phénomène du décrochage scolaire en Ontario français (Allaire, 2005; Allaire et al., 2005, Boissonneault, Michaud, Côté, Tremblay et Allaire, 2007; Michaud, Boissonneault, Côté, Tremblay et Allaire, 2008; Tremblay, Côté, Boissonneault, Michaud et Allaire, 2007; Tremblay, 2007) qui confirment l'ampleur du problème, mais qui offrent très peu d'explications quant au processus du choix scolaire. Effectivement, Allaire, Michaud, Boissonneault, Côté et Pounthioun ont souligné à la fin de leur rapport de 2005 qu'il est impératif "que l'on fasse une étude plus approfondie du phénomène du décrochage culturel pour en mieux connaître les causes et les effets » (p. 53).

Les propos qui précèdent montrent que le décrochage culturel s'avère un concept encore relativement peu défini dont la validité reste à démontrer. Ceci est d'autant plus impératif puisqu'il fait déjà l'objet de controverse. En 
effet, Lamoureux (2007) se penche sur la question de transition de l'école secondaire à l'université et examine le choix des élèves francophones de la douzième année concernant la fréquentation d'un établissement postsecondaire. Cette étude démontre que le choix des élèves de fréquenter une université de langue anglaise n'est pas un « décrochage » de la francophonie. Au contraire, la chercheure souligne "l'importance de la capacité de l'étudiant à pouvoir reconstituer son capital social et culturel pour mettre à profit son capital linguistique » (p. 286) dans la poursuite de ses études. II ne faut donc pas oublier que les jeunes Franco-Ontariens maîtrisent souvent deux langues ou plus et qu'en choisissant une langue de scolarisation, ils ne rejettent pas forcément leurs autres langues.

En somme, devant la nouveauté du concept de décrochage culturel, son association à celui de décrochage scolaire et le flou qui entoure sa définition, particulièrement en termes de causes et effets, de même que la controverse qu'il suscite déjà, il est permis de se demander quelle est la pertinence et l'apport de ce concept dans la compréhension du processus de choix scolaire que vivent les jeunes en milieu minoritaire francophone au Canada. Un examen plus approfondi de ce processus s'impose donc ici.

\section{Le processus de choix scolaire}

Au cours des dernières décennies, des changements importants ont été introduits dans les politiques concernant l'accès aux écoles publiques ${ }^{1}$. L'école du quartier n'est plus la seule option possible, parents et élèves pouvant, du moins en théorie, choisir l'école qui leur convient le mieux. Ce phénomène suscite des recherches autant au niveau national qu'international, de même que dans les milieux minoritaires francophones où le processus de choix se complexifie étant donné l'ajout des dimensions linguistique et culturelle. Les facteurs en jeu dans ce processus se regroupent en deux catégories principales selon qu'ils concernent le choix effectué par les parents ou celui effectué au niveau de la famille, incluant l'enfant.

\section{Processus de choix parental}

\section{L’impact du statut socioéconomique}

Sur le plan international, la littérature dans le domaine du choix scolaire des parents indique que le statut socioéconomique joue un rôle important dans ce processus (Bagley et Woods, 1998; Bagley, Woods et Glatter, 2001; Bosetti, 2004; Bulman, 2004; Davies et Aurini, 2008; Gewirtz, Ball et Bowe, 1995; Reay et Ball, 1998; Reay et Lucey, 2000). Les études révèlent deux catégories de parents : les participants au marché, qui sont informés et qui évaluent les différentes écoles possibles avant de choisir la meilleure option, et les non-participants, ceux qui ne sont pas informés et qui envoient leurs enfants à l'école assignée dans leur zone de recrutement (Bosetti, 2004; Bulman, 2004; Gewirtz et al., 1995) ou qui choisissent de ne pas s'investir dans le marché. On indique une corrélation importante entre le niveau d'éducation des parents et le revenu financier plus élevés chez ceux qui choisissent de façon active les écoles (Bosetti, 2004; Gewirtz et al., 1995). Les parents moins motivés à faire un choix scolaire sont plus souvent de la classe ouvrière (Bosetti, 2004; Reay et Ball, 1998). Ceci est expliqué par un sentiment d'infériorité chez ces derniers

\footnotetext{
${ }^{1}$ Le choix d'une école de langue française ou anglaise existe dans plusieurs municipalités ontariennes depuis les années 1980. Depuis l'approbation du financement des écoles secondaires catholiques de l'Ontario (1984) ainsi que la création des conseils scolaires de langue française (1998), de plus en plus de régions ont le choix entre écoles publiques et catholiques, tant francophones qu'anglophones, ainsi que l'option d'une école privée ou indépendante.
} 
parents puisque plusieurs d'entre eux « semblent se sentir incompétents dans le processus de choix, tout comme ils se sentent dans leurs autres relations avec l'école»(Reay et Ball, 1998, p. 433, traduction libre). Ce sentiment d'infériorité pourrait également provenir du manque de ressources disponibles pour les parents de la classe ouvrière.

En effet, la quantité et la qualité de capital social disponible sont directement liés au revenu des parents (Bagley et Woods, 1998; Bagley, Woods et Glatter, 2001; Bosetti, 2004; Bulman, 2004; Davies et Aurini, 2008; Reay et Ball, 1998; Reay et Lucey, 2000). L'accès à un cercle social plus informé constitue une ressource importante qui permet aux parents d'être bien équipés lorsqu'ils font leurs choix (Bosetti, 2004; Reay et Lucey, 2000). Bosetti (2004) soutient que l'égalité d'accès à l'école implique l'égalité d'accès : «à une information véridique et de qualité en ce qui a trait aux options de choix possibles, de même qu'à une information qui aide les parents à mieux comprendre les besoins éducatifs et les préférences de leur enfant» (p. 400, traduction libre). Étant donnée l'inégalité d'accès à l'information qui se révèle en fonction de la classe sociale des parents, les choix des parents de statut socioéconomique inférieur seraient plus problématiques, possiblement en raison du manque d'information à leur disposition.

Reay et Ball (1998) notent une autre différence selon le statut socioéconomique des parents. Il s'agit de leurs priorités quant au bonheur de leur enfant. Selon ces auteurs, les parents de la classe ouvrière s'intéressent davantage à ce que leurs enfants soient heureux à l'école au moment où ils la fréquentent. Par contre, les parents de la classe moyenne font plutôt référence au bonheur de leurs enfants dans le futur. En conséquence, Lucey et Reay (2002) remarquent que les parents de la classe moyenne vivent plus d'anxiété que d'autres lors du processus de choix scolaire, surtout en raison d'une importance exagérée accordée au choix de la « bonne » école pour leurs enfants.

En fin de compte, les différences selon le statut socioéconomique énumérées jusqu'ici pourraient avoir des conséquences importantes. Goldhaber (1999) fait remarquer que si seulement les familles de la classe moyenne exercent vraiment leur droit de choisir une école de façon éclairée, tel que la littérature le suggère (Bagley et Woods, 1998; Bagley, Woods et Glatter, 2001; Bosetti, 2004; Bulman, 2004; Davies et Aurini, 2008; Reay et Ball, 1998; Reay et Lucey, 2000), on pourrait voir encore plus de ségrégation raciale et économique dans les écoles. D'où l'intérêt de mieux comprendre ce phénomène en milieu minoritaire francophone.

Les recherches effectuées en milieu minoritaire au Canada s'attardent également au statut socioéconomique comme facteur qui influence le choix scolaire (Dalley et Saint-Onge, 2008; Deveau, Landry et Allard, 2006; Réseau CIRCUM, 1999). Dans leur recherche au Yukon, Dalley et Saint-Onge (2008) constatent que le choix scolaire des couples exogames, c'est-à-dire composés d'un parent francophone et d'un parent anglophone, est lié au statut socioéconomique : les parents des milieux professionnels choisissent plus souvent l'école francophone. Pour leur part, à l'instar de nombreux chercheurs sur le plan international (Bagley et Woods, 1998; Bagley et al., 2001; Bosetti, 2004; Bulman, 2004; Reay et Ball, 1998; Reay et Lucey, 2000), Deveau et al. (2006) précisent que la quantité de ressources disponibles pour les parents, et alors leur niveau de «naïveté sociale », est directement reliée au degré de scolarisation et à la profession que ceux-ci exercent. Les parents de la classe moyenne auraient donc un niveau de « naïveté sociale » moins élevé que les parents de classe inférieure. Ces chercheurs ont également constaté que le degré de scolarisation des parents est lié positivement au choix d'une école de langue française, car 75,5\% des 
parents qui choisissent des écoles secondaires de langue française ont terminé leurs études secondaires, comparativement à $41,6 \%$ de ceux qui optent pour la scolarisation en anglais, et à $43,8 \%$ de ceux qui optent pour une éducation bilingue de leurs enfants. Dans cette perspective, il semble important d'approfondir l'influence d'un autre facteur, notamment le consumérisme, dans le choix que font les parents pour l'école de leurs enfants.

\section{Les parents consommateurs et le magasinage d'écoles}

Les théoriciens en éducation utilisent souvent l'analogie du magasinage pour décrire le processus de choix d'écoles (Bagley et Woods, 1998; Bosetti, 2004; Davies et Aurini, 2008; Hirsch, 1994). D'après eux, l'expansion de l'idéologie du marché scolaire transforme «les parents en consommateurs et font de l'école un fournisseur de services; quant à l'élève, il est plus ou moins dans la même situation qu'une voiture en train d'être astiquée au garage » (Hirsch, 1994, p. 25). Suivant cette logique, Bosetti (2004) estime que les parents perçoivent le choix scolaire comme un processus de calcul des coûts, des bénéfices, des probabilités et des risques, afin de trouver la solution qui assurera le succès de l'enfant. Cette mentalité de consommateur alimente une nouvelle dynamique dans les relations entre parents et éducateurs. Davies et Aurini (2008) ont noté, au Canada, que cette mentalité « modifie les relations que les parents entretiennent avec les éducateurs des écoles publiques, passant d'un rôle de simple soutien à un rôle davantage de direction et même, de revendication » (p. 55, traduction libre). Cette dynamique a un impact sur la compétition entre les écoles de différents conseils scolaires, et parfois même, entre les écoles d'un même conseil scolaire.

La pratique du magasinage d'écoles s'appuie sur la théorie de la sélection sociale que mentionnent Bagley, Woods et Glatter (2001). Ces auteurs précisent que : « la sélection sociale implique que les parents choisissent l'environnement humain (élèves et personnel scolaire) en accord avec le type social (classe sociale, composition ethnique) qu'ils considèrent les plus appropriés ou les plus bénéfiques pour leur enfant » (p. 320, traduction libre). Cette théorie trouve aussi son application du côté des écoles qui font du marketing afin de recruter les élèves, une pratique nommée le « social targeting ». Le plus souvent, la cible visée est la population étudiante de la classe moyenne (Bagley et al., 2001; Hirsch, 1994) et la stratégie de recrutement privilégiée par les écoles se résume à se distinguer avec des taux supérieurs de réussite aux tests nationaux ou provinciaux, et aux autres évaluations importantes (Bagley et al., 2001; Bosetti, 2004; Davies et Aurini, 2008). Selon Bagley et Woods (1998), cette approche de compétition fait en sorte que les parents d'enfants en difficulté, ainsi que ces élèves eux-mêmes, sont souvent marginalisés dans cet environnement de concurrence. Prenant une perspective différente, Bagley et al. (2001) s'intéressent au rejet des écoles plutôt qu'au choix. Ces chercheurs concluent que les parents et les écoles peuvent se rejeter les uns et les autres. Effectivement, le choix scolaire revient souvent à un phénomène d'exclusion vécu par plusieurs familles puisque les « meilleures » écoles mettent à profit leur statut en rejetant de nombreux élèves de leur établissement pour en privilégier d'autres. Ces auteurs soutiennent que ces écoles travaillent davantage à améliorer leur image sur le marché scolaire plutôt qu'à réformer leurs programmes ou l'éducation qu'elles offrent. Comment cet environnement de compétition se manifeste-t-il en milieu minoritaire francophone?

Pour ce milieu en particulier, DiGiorgio (2006) étudie les stratégies de marketing utilisées par une école de langue française en Nouvelle-Écosse pour recruter ses élèves. L'auteure explique que : 
malgré l'existence du droit légal [re: Article 23], les directions et les parents d'écoles françaises doivent faire du marketing pour leurs écoles afin d'accroître leur clientèle. Les clients potentiels doivent être convaincus que l'école française est une meilleure option pour leurs enfants que les programmes d'immersion française ou anglais. (p. 93, traduction libre)

La chercheure souligne que l'école observée a recours à diverses méthodes de marketing, notamment la distribution de dépliants, la création d'un site internet et la rédaction d'articles dans les journaux. Le rôle des campagnes publicitaires visant à rehausser la perception d'une école par le public est considéré comme crucial dans le recrutement des élèves. Martel (2001) abonde dans le même sens en explorant les différentes façons d'attirer un plus grand nombre de familles vers le système d'éducation de langue française à travers le Canada avec des campagnes d'information pour les familles, la réforme de programmes interculturels et d'éveil aux langues, et l'organisation de structures et d'activités en langue française au sein des écoles ainsi que dans la communauté. Ces études montrent l'intérêt grandissant pour les stratégies de recrutement utilisées par les écoles de langue française en vue d'augmenter leurs effectifs scolaires. Cette compétition entre les écoles a-t-elle un impact sur la réussite scolaire des élèves?

\section{Une meilleure réussite pour tous les élèves?}

L'hypothèse selon laquelle la compétition entre les écoles améliore la qualité des institutions, de l'enseignement et, par conséquent, assure une meilleure réussite pour les élèves, revient souvent dans la littérature sur le choix scolaire (Card, Dooley et Payne, 2008; Davies et Aurini, 2008; Gewirtz et al., 1995; Goldhaber, 1999; Levin, 2002). En effet, Card et al. (2008), à la suite d'une recherche effectuée en Ontario, indiquent que lorsque les parents sont ouverts à la possibilité de changer d'écoles, les systèmes scolaires répondent d'une façon qui peut se traduire en une amélioration du succès de tous les élèves aux tests provinciaux. Leur comparaison des quartiers où plusieurs écoles sont en compétition avec des milieux ayant un nombre limité d'écoles et, par conséquent, un potentiel de compétition moins élevé, indique des améliorations notables lors des évaluations provinciales de troisième et de sixième années dans les régions plus concurrentielles. Cependant, Goldhaber (1999) et Gewirtaz et al. (1995) soulignent que la plupart des recherches s'attardent seulement aux tests et, alors, aux effets bénéfiques à court terme. II n'y a en effet, à notre connaissance, aucune étude qui s'intéresse au succès à long terme des élèves en relation avec la compétition entre écoles. En outre, selon certains, l'impact positif de la compétition n'est pas pour tous les élèves, mais se limite plutôt à ceux provenant des classes sociales plus élevées (Bagley et al., 2001; Bosetti, 2004). Bosetti (2004) conclut que la compétition existante s'adresse très peu aux problèmes liés à l'équité, à la diversité et à la cohésion sociale.

Pour leur part, les études recensées en milieu minoritaire francophone sont muettes sur la question de l'amélioration du succès scolaire des élèves. Selon cette littérature (Allard et al., 2004; Arsenault, 2008; Dalley et Saint-Onge, 2008; Deveau et al., 2006; Essiembre et al., 2005; Réseau CIRCUM, 1999; Tardif, 1995), la priorité chez les parents ayants-droit quant au succès de leurs enfants est soit la maîtrise de l'anglais, soit le développement d'un bilinguisme additif et équilibré, c'est-à-dire une forme de bilinguisme où l'apprentissage d'une nouvelle langue ne menace pas le maintien ou l'acquisition de la langue maternelle, comme nous en reparlerons plus loin. En outre, ces priorités de la part des parents semblent être directement liées à leur attachement culturel. 


\section{La place de la culture}

Plusieurs recherches notent l'influence remarquable de la culture sur le choix scolaire des parents (Allard, Essiembre et Arseneau, 2004; Arsenault, 2008; Bosetti, 2004; Bulman, 2004; Dalley et Saint-Onge, 2008; Deveau et al., 2006; English, 2009, Tardif, 1995). Cependant, les chercheurs ont des définitions différentes de la culture. Bosetti (2004) affirme que les valeurs et les croyances communes entre parents et école constituent un des facteurs les plus déterminants du choix scolaire chez les 1500 parents de son échantillon en Alberta. Selon cette chercheure, la culture est relative à l'ensemble des valeurs des parents. Dans le même sens, English (2009) s'inspire de Bourdieu et du concept de capital culturel. Cette chercheure met l'accent sur l'importance du développement du capital culturel à l'école. Elle précise que le fait pour l'école de promouvoir le "right» (p. 90) capital culturel en relation avec les valeurs différentes des parents est un facteur jugé prioritaire pour ces derniers lors du processus de choix scolaire. Les écoles australiennes faisant partie de son échantillon reconnaissent cette réalité et mettent en œuvre des programmes spécialisés en arts, en musique et dans certains sports comme stratégies de marketing, afin de promouvoir l'idée que leurs élèves auront ainsi une vie meilleure et deviendront exceptionnels.

Pour sa part, Bulman (2004), considère que « la culture doit être vue comme le tissu qui permet à chaque famille de donner du sens à l'éducation »( p. 493, traduction libre). II précise qu'on ne peut pas supposer que la culture est seulement l'ethnicité, le sexe, la classe sociale ou la religion d'une personne, mais qu'il s'agit plutôt des nombreux outils, ou le « tool kit », avec lesquels elle interagit dans sa vie quotidienne. Certains maintiennent que ces outils culturels renvoient à des motivations affectives et intrinsèques qui entrent en jeu lors du choix d'une école (Bagley et Woods, 1998; Bulman, 2004). De plus, malgré les différences en fonction de la classe sociale notées précédemment, la culture est un facteur important chez les parents, peu importe le statut socioéconomique (Bulman, 2004; English, 2009) et semble alors être un facteur universel quant au choix scolaire.

Pour ce qui est du discours déployé en milieu minoritaire francophone, plusieurs chercheurs ont établi que les facteurs culturels, qui incluent les valeurs communes au groupe minoritaire et le sentiment d'appartenance à la communauté francophone, sont parmi les plus influents lors du processus de choix scolaire (Arsenault, 2008; Dalley et Saint-Onge, 2008; Deveau et al., 2006; Essiembre et al., 2005; Réseau CIRCUM, 1999). Arsenault (2008) explique que l'appropriation des diverses cultures des parents par l'enfant est une priorité chez les parents exogames canadiens qu'elle a interviewé. Le choix de ces parents d'une école de langue française était basé en partie sur l'importance de la culture francophone. De même, Dalley et Saint-Onge (2008) notent que les parents de leur étude choisissent l'école francophone parce que la culture et les valeurs véhiculées par cette école cadrent avec les leurs. Au contraire, les parents ayants-droit qui choisissent un autre type d'école ne se reconnaissent pas dans la culture mise de l'avant par l'école francophone. Essiembre et al. (2005) ont également conclu que la motivation sous-jacente au choix d'une école de langue française pour les enfants des couples exogames était largement influencée par le désir de « transmettre la langue, la culture et l'identité françaises d'une génération à l'autre » (p. 2). En outre, SaintOnge (2002) affirme également que la culture est sans contredit le facteur le plus important en faveur du choix d'une école de langue française. 
L'appui accordé à la scolarisation en français de la part des parents, basé sur une culture définie en termes de construction identitaire et d'attachement à la communauté francophone, est également associé à des motivations plus affectives et intrinsèques que pragmatiques (Deveau et al., 2006; Réseau CIRCUM, 1999; Tardif, 1995). Deveau et al. (2006) indiquent que « le désir d'intégrer la communauté de langue française et l'identité ethnolinguistique francophone sont celles qui sont le plus fortement reliées à un positionnement favorable à la scolarisation en français » (p. 431). Les désirs personnels de faire partie de la communauté francophone sont alors des facteurs cruciaux lors du processus de choix. DiGiorgio (2006) explique que les écoles reconnaissent ce fait puisque la promotion de l'enrichissement du capital linguistique et culturel des élèves est une stratégie de marketing privilégiée par les écoles de langue française. Les chercheurs du Réseau CIRCUM (1999) soulignent aussi l'effet important que la culture pourrait avoir sur la rétention scolaire en affirmant que « toute politique qui aura pour conséquence d'accroître le sentiment de communauté francophone haussera les inscriptions dans les écoles françaises » (document en format HTML, sans page). Les écoles considèrent donc que la promesse d'un environnement propice à la construction identitaire pourrait largement augmenter leurs effectifs. Reconnaissant la valeur que les parents accordent au bilinguisme, les écoles soutiennent également que les jeunes atteindront un niveau de bilinguisme équilibré lorsqu'ils choisissent de fréquenter une école de langue française.

\section{Bilinguisme : une priorité pour les parents canadiens}

Alors qu'aucune étude issue de la littérature internationale n'aborde la question du bilinguisme, plusieurs études portant sur la minorité linguistique francophone du Canada précisent que l'appropriation du français et de l'anglais est une priorité dans le processus de choix scolaire pour les parents, étant donné les bénéfices économiques et sociaux associés à la connaissance des deux langues (Allard et al., 2004; Arsenault, 2008; Dalley et Saint-Onge, 2008; Deveau et al., 2006; Essiembre et al., 2005; Réseau CIRCUM, 1999; Tardif, 1995). Des parents d'Halifax, de Sudbury, de Calgary et de Vancouver dévoilent que le prestige associé au bilinguisme figure parmi les facteurs déterminants de leur choix (Réseau CIRCUM, 1999). Ce facteur peut aussi bien agir en faveur du choix d'une école française que d'une école anglaise. En effet, cette même étude révèle que les principales raisons fournies par les parents qui choisissent l'école anglaise, plutôt que l'école de langue française, sont associées à l'exogamie du couple et à la perception négative de l'école française au regard de l'apprentissage de l'anglais. Deveau et al. (2006) constatent également cette réalité. Les chercheurs du CIRCUM (1999) suggèrent même que « davantage de parents choisiront l'école française s'ils sont convaincus que leurs enfants maîtriseront la langue anglaise à la fin de leur scolarisation » (section 3.1). Dans un effort pour contrer cette tendance, Allard et al. (2004) précisent que le couple exogame qui désire que son enfant développe un bilinguisme équilibré devrait agir en fonction du principe selon lequel : « c'est en mettant l'accent sur la langue la plus faible ou fragile dans la région, c'est-à-dire le français, que le couple exogame s'assurera que son enfant développera un bilinguisme additif équilibré » (p. 12).

Pour leur part, les parents de l'échantillon de Dalley et Saint-Onge (2008) ont tous soulevé l'importance du bilinguisme pour leurs enfants, mais avec certaines nuances. Ils ne privilégient pas nécessairement une plus grande maîtrise du français aux dépens de l'anglais, et certains vont même jusqu'à favoriser une plus grande maîtrise de l'anglais. Le bilinguisme recherché n'est donc pas dans tous les cas celui de type « équilibré » prôné par Allard et al. 
(2004) et les écoles de langue française. Ces chercheures ont également observé que le choix de l'école anglophone ne représente pas un rejet de la langue française de la part des parents ou des enfants puisque tous les parents francophones en union exogame désirent que leurs enfants soient bilingues.

En fin de compte, les choix scolaires des parents francophones et acadiens nés au Canada font suite à des considérations similaires et dissemblables des parents au niveau international. Le consumérisme et le magasinage des écoles, la compétition entre écoles et l'impact du statut socioéconomique viennent influencer tous les parents quant au phénomène de choix d'une école. Par ailleurs, la culture vient également influencer l'ensemble des parents, mais l'importance et la définition de celle-ci diffèrent selon les auteurs. Le facteur qui prime pour les parents anglophones et francophones à la fois revient au bilinguisme. La question linguistique semble alors centrale dans le processus de choix scolaire des parents, mais elle n'est surtout pas la seule considération. En effet, l'opinion de l'élève peut également avoir une portée dans la décision de choisir une école, surtout lorsqu'il s'agit de choisir une école secondaire. La prochaine section discute donc du rôle des jeunes dans le processus de choix scolaire.

\section{Le choix scolaire familial, incluant la participation des enfants}

Beaucoup moins d'études s'attardent plus spécifiquement au processus de choix scolaire tel qu'il est vécu par la famille, incluant les enfants. Ces études révèlent que malgré l'influence incontestable des parents, les jeunes se fient abondamment à l'opinion de leurs amis et de leurs pairs, et semblent valoriser les facteurs linguistiques et culturels d'une manière différente de leurs parents.

\section{L'influence incontestable des parents}

Quelques études recensées en provenance de divers pays traitent du choix d'une école secondaire comme un processus familial qui inclut les enfants et les parents (Hirsch, 1994; Lucey et Reay, 2002; Reay et Ball, 1998; Reay et Lucey, 2000; Tardif, 1995). En France, Hirsch (1994) conclut que le degré de participation des enfants au choix est surtout une question d'âge : « dès l'âge de 11 ans, les élèves choisissent sur un pied d'égalité avec leurs parents, et [...] à partir de 14 ans, leur avis commence à prédominer, surtout lorsque le choix de l'école détermine celui du programme » (p. 26). Cette conclusion, si appliquée en Ontario, suggère que les jeunes seraient davantage responsables du choix d'une école secondaire. En effet, selon les données du Ministère de l'éducation de l'Ontario (2006), les jeunes effectuent leur choix scolaire lors de la transition de l'école primaire à l'école secondaire à l'âge de 13 ou 14 ans.

Qui plus est, deux études concluent que l'influence de l'enfant sur le choix scolaire d'une école secondaire est largement déterminée par le statut socioéconomique des parents (Reay et Ball, 1998; Reay et Lucey, 2000). D'après ces études menées en Grande-Bretagne, la majorité des parents de la classe ouvrière laissent leur enfant choisir son école. Reay et Ball (1998) précisent que ceci est dû, encore une fois, au sentiment d'infériorité ressenti par ces parents face au monde de l'éducation et au fait qu'ils considèrent leur enfant comme l'expert éducationnel de la maison. Les jeunes interviewés par Reay et Lucey (2000) confirment la prédominance de leurs propres choix sur ceux de leurs parents. Au contraire, la majorité des parents de classe moyenne prennent ultimement la décision finale quant à l'école secondaire fréquentée par leur enfant. Plusieurs parents interviewés par Reay et Ball (1998) soulignent 
l'importance des opinions de leur enfant et de la discussion ouverte, mais ils sont d'avis que leur choix est le meilleur, même s'il n'est pas en accord avec celui de l'enfant. Toutefois, le haut niveau d'implication de certains parents, leur rôle étant parfois dominant, peut avoir des conséquences pour leurs enfants. Lucey et Reay (2002) remarquent que le processus de choix scolaire lors de la transition de l'école primaire à l'école secondaire peut être une source d'anxiété chez les jeunes. Les chercheures concluent que l'anxiété et le stress que vivent les élèves de leur échantillon sont causés par les nombreuses attentes de la part des parents qui veulent à tout prix qu'ils fréquentent la bonne école.

En milieu minoritaire francophone canadien, très peu d'études mentionnent le choix scolaire comme étant un processus familial. Tardif (1995) a mené une enquête auprès d'élèves de ge et 10e années de trois écoles francophones de l'Alberta et a également contacté leurs parents pour mieux comprendre le processus familial du choix scolaire. Elle conclut que les parents, indépendamment de la classe sociale, ont une influence capitale sur le choix scolaire de leurs enfants. Le jugement des parents quant à l'éducation en français et même, à l'importance de la langue française, est primordial puisque les enfants optent plus souvent pour l'école anglophone lorsque leurs parents ne croient pas à l'importance de fréquenter une école de langue française. Les priorités des parents quant au développement du bilinguisme de leurs enfants jouent alors un rôle crucial.

Dans notre propre recherche de maîtrise, les résultats du sondage effectué auprès de 79 élèves de $8^{\mathrm{e}}$ année d'une école française en milieu minoritaire ontarien, révèlent que les élèves se disent également partagés quant à l'influence de l'opinion de leur père, $50 \%$ des répondants indiquant aucune ou peu d'influence, l'autre $50 \%$, assez ou beaucoup d'influence. La réponse la plus populaire, « aucune influence », a été choisie par $33 \%$ des répondants. L'influence de l'opinion de la mère se révèle plus forte : $66 \%$ des répondants ont indiqué que celle-ci avait assez ou beaucoup d'influence. Une hypothèse pouvant expliquer ces résultats pourrait tenir au fait que plus de mères que de pères de notre échantillon avaient le français comme langue première. Le rôle de la mère dans la transmission de la langue et dans la promotion de l'école de langue française est donc à prendre en compte.

Ces données montrent une variation importante quant à l'influence des parents selon les élèves eux-mêmes. Même si plus de la moitié d'entre eux sont influencés par leurs parents, il faut toujours se demander comment l'autre moitié des élèves arrive à prendre sa décision. Quels sont donc les autres facteurs d'influence?

\section{Une priorité importante pour les élèves : les amis et les pairs}

Bien que les parents aient une influence notable, les élèves eux-mêmes peuvent être impliqués de façon significative lors du choix scolaire et leurs priorités ont un impact sur ce choix (Reay et Lucey, 2000). La littérature suggère que lorsqu'un élève choisit son école secondaire, la prise en compte du choix des amis et des pairs a une influence non négligeable (Bagley, Woods et Glatter, 2001; Bulman, 2004; Reay et Ball, 1998; Reay et Lucey, 2000). Les chercheurs notent plusieurs références, peu importe la classe sociale, aux préoccupations des élèves quant aux choix d'écoles de leurs amis (Bulman, 2004; Reay et Ball, 1998). Une raison importante pour rejeter une école revient également aux amis qui fréquentent ou qui ne fréquentent pas cet établissement (Bagley et al., 2001). De plus, les élèves peuvent influencer leurs amis lors des discussions entre pairs sur le choix scolaire (Reay et Lucey, 2000). L'aspect social du choix scolaire est donc présent de plusieurs façons différentes. 
Les données recueillies dans notre enquête semblent corroborer ces constats étant donné que $61 \%$ des jeunes rapportent que leurs amis ont eu assez ou beaucoup d'influence sur leur choix. Ce résultat révèle que le rôle des pairs dans le processus de choix scolaire est proportionnellement très important et que ce facteur doit être pris en considération dans toute intervention visant à influencer ce choix. Pour que les élèves mettent à profit leur capital social lors du processus de choix scolaire, il devient important de s'assurer que leur cercle social, c'est-à-dire leurs amis et leurs pairs, leur fournissent les informations pertinentes pour un choix éclairé.

\section{Facteurs linguistiques et culturels}

Par ailleurs, en milieu minoritaire francophone, les résultats de l'étude de Tardif (1995) indiquent que les élèves ayant quitté l'école de langue française ont moins de confiance en leur maîtrise du français à l'oral et à l'écrit que ceux qui choisissent de poursuivre leurs études au niveau secondaire dans le système francophone. Les élèves préfèrent poursuivre leurs études dans la langue où ils se sentent plus à l'aise à l'oral et à l'écrit. L'insécurité linguistique en français semble donc causer des difficultés particulières chez certains jeunes et les amène à s'orienter vers un environnement dans lequel leur niveau de sécurité linguistique est plus élevé.

Dans le cadre de notre sondage, les élèves ont indiqué les facteurs linguistiques qu'ils considèrent les plus influents lors du processus de choix scolaire. L'importance de maîtriser l'anglais est reconnue par 73 \% des répondants, alors que $80 \%$ d'entre eux expriment une opinion semblable pour le français. Lorsque la question est élargie au-delà du choix d'une école pour envisager l'ensemble du futur de l'élève, $83,5 \%$ des répondants affirment l'importance de l'anglais et $90 \%$, l'importance du français. Puisque $15 \%$ des répondants ont choisi une école secondaire de langue anglaise, il semble que même un certain nombre d'entre eux reconnaissent l'importance du français pour leur futur. Étant donné que l'enquête a été menée en milieu extrêmement minoritaire, il s'avère d'un intérêt particulier de constater qu'un pourcentage très élevé d'élèves reconnait l'importance de la langue minoritaire pour le futur, un futur qui pourrait inclure des études postsecondaires, une carrière ou élever des enfants.

Lorsque questionnés sur la culture elle-même, les élèves ciblés dans notre enquête ne semblent pas motivés par les mêmes facteurs culturels que leurs parents. En effet, $63 \%$ des répondants considèrent que la culture n'a aucune ou peu d'influence sur leur choix. Bien que les facteurs linguistiques semblent avoir énormément d'influence sur les élèves, la culture ressort comme étant le facteur qui les rejoint le moins, alors qu'elle se révèle être l'un des plus importants pour les parents. La question se pose alors à savoir quelles représentations les élèves se font de la culture, et en quoi ces représentations diffèrent de celles de leurs parents.

En conclusion, le degré d'influence des parents sur le choix des élèves semble être déterminé par plusieurs facteurs : l'âge de l'élève, le statut socioéconomique de la famille, l'opinion des parents quant à l'importance de la langue française et du bilinguisme. Du point de vue des élèves, l'influence des parents, en particulier celle de la mère, semble le facteur décisif pour la moitié d'entre eux. Pour les autres, l'opinion des amis et des pairs, peu importe la classe sociale, semble privilégiée. Quant à la langue, deux facteurs se révèlent déterminants, l'insécurité linguistique et l'importance du bilinguisme pour le futur des élèves à court et à long terme. Qui plus est, les élèves accordent beaucoup moins d'importance à la culture qu'à la langue lorsqu'ils vivent le processus de choix d'une école. 
Ainsi, après avoir passé en revue les multiples facteurs impliqués dans le processus de choix scolaire, la question de la pertinence de l'expression « décrochage culturel » est alors de premier ordre.

\section{La pertinence du concept de « décrochage culturel »}

Comme il a été précisé au début de cet article, le concept de décrochage culturel s'avère relativement peu défini, faisant simplement référence au fait qu'un élève ayant complété sa scolarité primaire en français choisit de poursuivre ses études secondaires dans une école de langue anglaise. Associé au phénomène du décrochage scolaire, ce concept prend une valeur négative, et peut ainsi contribuer à la stigmatisation des élèves qualifiés de décrocheurs culturels. La littérature sur le processus de choix scolaire supporte-t-elle un tel concept?

Une première constatation qui se dégage de la recension des écrits est le désir croissant des parents et des élèves d'exercer leur droit de choisir leur école, et pas n'importe quelle école, mais la « bonne » école qui leur assurera les meilleures chances de succès dans le futur. Déjà, cette constatation remet en cause la dimension « décrochage » du concept de « décrochage culturel ». En effet, la très grande majorité des facteurs en jeu dans le processus de choix d'une école secondaire, même s'ils diffèrent quelque peu selon qu'on considère le point de vue des parents ou celui des élèves, n'impliquent pas nécessairement le désir de s'éloigner d'une situation jugée insatisfaisante. En fait, seulement deux facteurs semblent suggérer un décrochage. Le premier concerne la valeur associée au bilinguisme par les parents. En effet, on rapporte que certains parents s'orientent vers l'école anglaise lorsqu'ils sont convaincus que celle-ci contribuera au développement du type de bilinguisme qu'ils envisagent pour leur enfant plus que ne l'a fait auparavant l'école francophone. Le deuxième facteur se révèle dans l'insécurité linguistique en français ressentie par un certain nombre d'élèves, ce qui les amène à s'orienter vers une école anglaise plus sécurisante au niveau secondaire. Ces deux facteurs sont-ils suffisants pour justifier l'emploi du terme « décrochage »?

Si l'on examine la deuxième dimension du concept, l'emploi du qualificatif « culturel » suggère que la culture est conçue dans un sens global qui renvoie à l'ensemble des facteurs conduisant au choix d'une école secondaire de langue autre que le français. Cependant, les écrits recensés ne semblent pas supporter une telle conception de la culture. En effet, on constate que le consumérisme et le magasinage d'écoles, la compétition entre écoles et le statut socioéconomique sont susceptibles d'influencer tous les parents dans le processus de choix d'une école. L'ensemble de ces facteurs alimente la compétition entre écoles et résulte dans une variété plus riche de choix possibles pour les familles du XXle siècle. Mais ces facteurs, aussi importants soient-ils, semblent associés davantage à des motivations pragmatiques plutôt qu'aux motivations affectives et intrinsèques reliées à la culture, du moins selon les définitions proposées par les auteurs consultés. Ceci s'applique également à la valeur accordée au bilinguisme. Les multiples recherches qui touchent à la culture révèlent que la transmission de l'héritage culturel est une priorité incontestable lors du choix d'une école, et cela se voit sur le plan international tout comme en milieu minoritaire. Cependant, peu de recherches s'attardent à examiner quelles sont véritablement les motivations culturelles variées, regroupées sous les étiquettes de construction identitaire et d'attachement à la communauté. Comment peut-on expliquer, par exemple, que certains parents ne se reconnaissent pas dans la culture de l'école? En outre, les recherches négligent d'envisager l'expérience culturelle des enfants. À quoi peut-on attribuer le fait que plus des deux tiers des élèves ayant 
participé à notre sondage, et dont la majorité choisit de poursuivre des études dans une école de langue française, considèrent que la culture a peu ou pas d'influence sur leur choix scolaire? Ce résultat suggère que les facteurs culturels qui entrent en jeu lors du processus de choix d'une école sont fort complexes et qu'il conviendrait de repenser le rapport de l'école à la culture en prêtant une plus grande attention au point de vue des parents tout autant qu'à celui des élèves.

En somme, en révisant les nombreux facteurs qui influencent le choix des parents et des jeunes, l'on constate que la culture apparaît comme un facteur parmi d'autres. L'on ne peut donc pas réduire le choix d'une école de langue anglaise à un choix uniquement culturel. En conséquence, si un élève quitte l'école de langue française pour une école de langue anglaise, que ce soit un choix parental, familial ou individuel, il ne s'agit pas nécessairement d'un décrochage « culturel ». L'utilisation de ce qualificatif s'apparente à une généralisation à outrance, non supportée par les données empiriques.

Pour compléter cette analyse, il convient d'attirer l'attention sur les effets pervers de la prolifération dans l'usage de l'expression « décrochage culturel ». Lamoureux (2007) souligne que le modèle de l'élève idéal véhiculé par l'école de langue française nécessite que l'élève poursuive ses études en français. Alors, les finissants d'écoles de langue française de son échantillon qui choisissent des établissements postsecondaires de langue anglaise ressentent qu'ils doivent toujours justifier ce choix et manifestent une certaine culpabilité face à celui-ci. Certains chercheurs semblent alimenter cette culpabilité par des affirmations à l'effet que, par exemple, « le fait de poursuivre [des études] en anglais compromet sérieusement [la] capacité de fonctionner en français et certainement de nourrir la communauté en français » (Frenette et Quazi, 1997, p. 3). Labrie (2007) attire l'attention sur le fait que l'utilisation de l'expression " décrocheurs culturels » suggère qu'il s'agit d'un "problème dont les jeunes seraient responsables et qui compromettrait leur propre avenir » (p. 10), ce qui n'est vraisemblablement pas le cas. Ces jeunes portent un jugement sur le système scolaire de langue française et choisissent en fonction de leurs capacités et préférences linguistiques, leurs intérêts et leurs buts postsecondaires. Labrie (2007) pose alors la question : « ne devrait-on pas plutôt parler de la difficulté de l'école de langue française de retenir et d'attirer les élèves de langue française? Ce serait déplacer la responsabilité du jeune vers l'institution » (p. 10).

Tous n'utilisent pas nécessairement les expressions décrochage culturel ou décrocheur culturel de la même façon, mais il importe de souligner que leur emploi peut avoir des conséquences négatives pour les élèves concernés et pour la communauté francophone. Une partie des élèves sondés dans le cadre de notre projet de recherche, et qui ont choisi une école secondaire de langue anglaise, ont tout de même indiqué qu'ils prévoient inscrire leurs enfants à une école de langue française. Puisque la culture n'était pas un facteur déterminant pour la plupart des participants, il est permis de croire qu'ils ne rejettent pas leur culture francophone et qu'ils veulent continuer à faire partie de la communauté franco-ontarienne. En utilisant le terme décrocheur culturel, on court le risque d'exclure ou même, d'ostraciser ces élèves.

\section{Conclusion}

Le choix d'un mot ou d'une expression pour désigner une réalité n'est pas sans conséquence. Dans la perspective bourdieusienne mentionnée précédemment, les mots sont non seulement chargés de signification, mais ils 
peuvent même concourir à l'exercice du pouvoir sur l'autre. Ainsi, nous pouvons constater que l'utilisation de l'expression décrochage culturel s'est répandue dans le domaine de l'éducation en Ontario français au cours des dernières décennies. Cependant, la définition de celle-ci est toujours imprécise et ne permet pas de vraiment cerner la réalité qu'elle est censée représenter. De plus, dans l'état actuel des connaissances sur le processus de choix scolaire, nous ne pouvons pas affirmer avec certitude que le choix d'une école dont la langue d'instruction est autre que le français est attribuable uniquement à l'insatisfaction face à la culture transmise à l'école française. En effet, la recension des écrits indique que plusieurs autres facteurs tels que, le statut socioéconomique de la famille, le consumérisme, l'opinion des parents quant à l'importance de la langue française et du bilinguisme, l'influence des parents en fonction de l'âge de l'élève, l'opinion des amis et l'insécurité linguistique viennent influencer soit les parents, les enfants, ou les deux, lorsqu'ils s'apprêtent à choisir la langue d'instruction et l'école secondaire. Ce qui importe, pourtant, ce sont les conséquences possibles de l'emploi de l'étiquette « décrocheur culturel » pour désigner les élèves qui font un tel choix : culpabilisation, discrimination et à la limite, exclusion. Cela étant dit, il convient de préciser que l'analyse critique des expressions décrochage culturel et décrocheur culturel dans le cadre de cet article vise à alimenter la réflexion par rapport à leur utilisation. Dans l'état actuel des connaissances et faute de réflexion plus approfondie, il est permis de recommander que la prudence demeure de mise.

Cet article fait état d'une recension d'écrits provenant du Canada anglophone et de plusieurs autres pays, et elle inclut également des études qui présentent la réalité spécifique du milieu minoritaire francophone au Canada. Bien qu'il existe plusieurs rapports de recherches menées dans ce dernier milieu (Allard et al., 2004; Arsenault, 2008; Bosetti, 2004; Deveau et al., 2006; Dalley et Saint-Onge, 2008; Lamoureux, 2007; Martel, 2001; Réseau CIRCUM, 1999; Tardif, 1995), seulement deux d'entre eux traitent spécifiquement du choix scolaire en Ontario (Lamoureux, 2007; Martel, 2001). Ce manque d'études menées en Ontario constitue une lacune importante qui met en évidence l'urgence d'étudier ce phénomène davantage au niveau de la province afin d'avoir accès à des données plus précises pour guider les écoles, les parents et les élèves lors du processus de choix scolaire. II importe de développer une compréhension élargie des facteurs reliés au positionnement des parents et des enfants envers la langue de scolarisation et le choix d'une école afin que le personnel scolaire puisse mieux ajuster ses interventions aux valeurs, aux préoccupations et aux attentes de la communauté, de même qu'élaborer des stratégies de sensibilisation et de recrutement plus efficaces afin d'augmenter la rétention scolaire dans les écoles de langue française.

\section{Références}

Allaire, G. (2005). De la théorie à la pratique : Le décrochage scolaire en Ontario français : le point de vue des jeunes... <http://www.edu.gov.on.ca/fre/teachers/studentsuccess/monographie3.pdf>

Allaire, G., Michaud, J., Boissonneault, J., Côté, D. et Pounthioun, D. (2005). Le décrochage au secondaire en Ontario français : le point de vue des jeunes. Rapport présenté à la Direction des politiques et des programmes d'éducation en langue française du ministère de l'Éducation de l'Ontario. Sudbury : Institut franco-ontarien, Université Laurentienne.

Allard, R., Essiembre, C. et Arseneau, S. (2004). Les valeurs et les choix du couple exogame. Dans G. Taylor (dir.). ConnEXions, Publication pour les familles exogames, Edmonton, Fédération des parents francophones de l'Alberta, 10-15.

Arsenault, L. (2008). Le choix de l'école francophone par une famille exogame : exemple d'une approche sociolinguistique et ethnographique. Dans P. Dalley et S. Roy (dir.). Francophonie, minorités et pédagogie (p. 259-280). Ottawa : Presses de I'Université d'Ottawa.

Bagley, C. et Woods, P.A. (1998). School Choice, Markets and Special Educational Needs. Disability \& Society, 13(5), 763-783.

Bagley, C., Woods, P.A. et Glatter, R. (2001). Rejecting schools : Towards a fuller understanding of the process of parental 
choice. School Leadership and Management, 21(3), 309-325.

Boissonneault, J., Michaud, J., Côté, D., Tremblay, C.et Allaire, G. (2007). L'abandon scolaire en Ontario français et perspectives d'avenir des jeunes. Éducation et francophonie, 35(1), 3-22.

Bosetti, L. (2004). Determinants of school choice : Understanding how parents choose elementary schools in Alberta. Journal of Education Policy, 19(4), 387-405.

Bourdieu, P. (1982). Ce que parler veut dire. Paris : Fayard.

Bourdieu, P. et Passeron, J-C. (1970). La reproduction : éléments pour une théorie du système d'enseignement. Paris : Éditions de Minuit.

Bulman, R. C. (2004). School choice stories : The role of culture. Sociologicial Inquiry, 74(4), 492-519.

Card, D., Dooley, M. et Payne, A. (2008). School Competition and Efficiency with Publicly Funded Catholic Schools. Working Paper No. 14176. Cambridge : National Bureau of Economic Research.

Dalley, P. et Saint-Onge, H. (2008). Choix scolaire des couples exogames au Yukon. Dans

P. Dalley et S. Roy (dir.). Francophonie, minorités et pédagogie (p. 121-142). Ottawa : Presses de l'Université d'Ottawa.

Davies, S.et Aurini, J.D. (2008). School Choice as Concerted Cultivation : the case of Canada. Dans M. Forsey, S. Davies et G. Walford (dir.), The Globalisation of School Choice? (p. 55-71). Oxford : Symposium Books.

Deveau, K., Landry, R. et Allard, R. (2006). Facteurs reliés au positionnement envers la langue de scolarisation en milieu minoritaire francophone : le cas des ayants droit de la Nouvelle-Écosse (Canada). Revue des sciences de l'éducation, 32(2), 417-437.

DiGiorgio, C. (2006). What Happens After the Law is Passed? Marketing to Parent Demands in a Canadian Francophone School. Journal of School Choice, 1(3), 91-113.

English, R. (2009). Selling Education Through 'Culture': Responses to the Market by New, Non-Government Schools. The Australian Educational Researcher, 36(1), 89-104.

Essiembre, C., Arseneau, S. et Allard, R. (2005). Expériences et besoins des ayants droit et de leurs enfants d'âge préscolaire concernant la transmission de la langue et de la culture, le choix de la langue d'éducation et les services offerts à la petite enfance du Nouveau-Brunswick. Moncton: Centre de recherche et de développement en éducation (Université de Moncton).

Fédération de la jeunesse franco-ontarienne (FESFO) (1993). Mémoire présenté à la Commission royale sur l'éducation. Toronto : Gouvernement de l'Ontario.

FESFO (2006). Jeunesse en développement économique communautaire. <http://www.ccednet-rcdec.ca/files/ccednet/ FESFO_Ultimate_Final_FR.pdf>

Ferguson, B., Tilleczek, K. et Rummens, J. A. (2005). Early School Leavers : Understanding the Lived Reality of Student Disengagement from Secondary School. A Final Report. Toronto : Community Health Systems Resource Group, The Hospital for Sick Children of Toronto.

Frenette, N. et Quazi, S. (1997). L'effectif et la rétention des élèves dans les écoles françaises en situation minoritaire au Canada 1980-1994. Winnipeg : La Commission nationale des parents francophones.

Gewirtz, S., Ball, S. J. et Bowe, R. (1995). Markets, Choice and Equity in Education. Buckingham : Open University Press.

Goldhaber, D. (1999). School choice : an examination of the empirical evidence on achievement, parental decision making and equity. Educational Researcher, 28(9), 16-25.

Hirsch, D. (1994). Processus du choix d'une école: profils, contraintes et influences des politiques. Dans D. Hirsch (dir.). L'école : une affaire de choix (p. 25-38). Paris : Organisation de Coopération et de Développement Économiques.

Labrie, N. (2007). La recherche sur l'éducation de langue française en milieu minoritaire : pourquoi? Dans Y. Herry et C. Mougeot (dir.). Recherche en éducation en milieu minoritaire francophone (p. 1-14). Ottawa: Presses de l'Université d'Ottawa.

Lamoureux, S. A. (1995). Du décrochage culturel à la persistance scolaire à l'école franco-ontarienne (mémoire de maîtrise inédit). Toronto : Université de Toronto.

Lamoureux, S. A. (2007). La transition de l'école secondaire de langue française à l'université en Ontario : perspectives étudiantes (thèse de doctorat inédite). Toronto : Université de Toronto.

Lucey, H. et Reay, D. (2002). Carrying the beacon of excellence : social class differentiation and anxiety at a time of transition. Education Policy, 17(3), 321-336.

Martel, A. (2001). Droits, écoles, et communautés en milieu minoritaire : 1986-2002. Analyse pour un aménagement du français par l'éducation. Ottawa : Commissariat aux langues officielles.

Michaud, J., Boissonneault, J., Côté, D., Tremblay, C. et Allaire, G. (2008). Décrochage, raccrochage et rétention scolaires selon les jeunes franco-ontariens. Francophonies d'Amérique, 25, 125-155. 
Ministère de l'éducation de l'Ontario (2006). School Program Files, 1998-99 to 2004-05, Ontario School Information System (OnSIS), données inédites. Toronto : Ministère de l'éducation de l'Ontario.

Patrimoine canadien (2006). PLAN D'AFFAIRES 2005-2006 à 2008-2009. [Entente] Canada-Ontario 2005-2006 à 2008-2009 relative aux fonds complémentaires en appui à l'amélioration de l'éducation en langue française. <http://www.pch.gc.ca/pgm/lo-ol/entente-agreement/education/ont/2005-2007/business-plan-fra.cfm>

Radio Canada (2011). Collège Boréal: contrer le décrochage culturel. < http://www.radio-canada.ca/regions/Ontario/ 2011/06/13/007-college-boreal-phase2.shtml >

Reay, D. et Ball, S. (1998). 'Making Their Minds Up': Family Dynamics of School Choice. British Educational Research Journal, 24(4), 431-448.

Reay, D. et Lucey, H. (2000). Children, School Choice and Social Differences. Educational Studies, 26(1), 83-100.

Réseau CIRCUM (1999). Motivations en ce qui a trait aux choix scolaires chez les parents ayants droit hors Québec : Rapport de recherche. Ottawa : Commissariat aux langues officielles. < http://circum.com/textes/motiv/motiv_f.htm>

Saint-Onge, H. (2002). Le choix scolaire des parents ayants droit de Whitehorse au Yukon (thèse de maîtrise inédite). Edmonton : Campus Saint-Jean, University of Alberta.

Tremblay, C. (2007). « Décrochage culturel» au secondaire en Ontario français. Dans J. Boissonneault et M. Bernier (dir.) Actes de la 13 e Journée Sciences et Savoirs (p.79-89). . Sudbury : Acfas-Sudbury.

Tremblay, C., Côté, D., Boissonneault, J., Michaud, J. et Allaire, G. (2007). Point de vue des jeunes sur le décrochage scolaire au secondaire en Ontario français. Dans J. Boissonneault et M. Bernier (dir.). Actes de la $13^{e}$ Journée Sciences et Savoirs (p. 231-244). Sudbury : Acfas-Sudbury. 